\title{
DENSITOMETRIA DE RAIOS X NAAVALIAÇÃO DA DENSIDADE EM PAINÉIS DE PARTÍCULAS
}

\section{X-RAY DENSITOMETRY IN THE EVALUATION OF DENSITY IN PARTICLEBOARD PANEL}

\author{
Fabricio Gomes Gonçalves ${ }^{1}$ Roberto Carlos Costa Lelis ${ }^{2}$ Alexandre Monteiro de Carvalho ${ }^{2}$ \\ Mário Tomazello Filho ${ }^{3}$
}

\begin{abstract}
RESUMO
Objetivou-se neste trabalho determinar o perfil da densidade aparente em painel aglomerado confeccionado com partículas da madeira de Acacia mangium. Utilizou-se a técnica de microdensitometria de raios X correlacionada com a densidade gravimétrica. Foram utilizados os adesivos ureia formaldeído e tanino comercial de Acacia mearnsii aplicado na forma de pó diretamente nas partículas previamente encoladas. Os painéis foram fabricados com quatro razões de compactação $(1,39 ; 1,55 ; 1,73 ; \mathrm{e}, 2,00)$ combinadas a quatro proporções de tanino em pó $(0,10,20$ e 30\%), totalizando 16 tratamentos (4 painéis por tratamento). Foram determinados, analisados e comparados o perfil da densidade aparente (densidade média, densidade máxima e densidade mínima) e a densidade gravimétrica dos aglomerados produzidos. O perfil da densidade é apresentado graficamente e interpretado por análise de variância e regressão linear múltipla. Os valores de densidade aparente média, máxima e mínima observados para os painéis aglomerados obtidos pela microdensitometria de raios X variaram de 357 a $697 \mathrm{~kg} / \mathrm{m}^{3}, 386$ a $824 \mathrm{~kg} / \mathrm{m}^{3}$ e 334 a $634 \mathrm{~kg} / \mathrm{m}^{3}$, respectivamente, e pelo método gravimétrico de 369 a $742 \mathrm{~kg} / \mathrm{m}^{3}$. A técnica mostrou-se eficiente na determinação de variações nos perfis de densidade aparente ao longo da espessura dos painéis em todos os tratamentos, bem como mostrou-se eficiente na obtenção direta da densidade aparente. Estas variações poderiam ser minimizadas se o tanino utilizado fosse anteriormente hidratado em água. Houve forte correlação entre as médias das densidades obtidas pelo método do microdensitômetro de raios $\mathrm{X}$ e pelo método gravimétrico.
\end{abstract}

Palavras-chave: qualidade de chapa; análise não destrutiva; perfil de densidade.

\section{ABSTRACT}

This study aimed to determine the apparent density, by gravimetric technique and X-ray microdensitometry of particleboard produced with Acacia mangium wood and search existence of correlation between both methods. It was used urea formaldehyde adhesive and commercial tannin Acacia mearnsii applied in powder form directly to the previously glued particles. The panels manufactured using four compression ratios $(1.39,1.55,1.73$, and 2.00) combined four levels of powdered tannin $(0,10,20$ and 30\%), making up 16 treatments ( 4 panels by treatment). The particleboard apparent density parameters (mean, maximum and minimum) and gravimetric density were determined, analyzed and compared. The density profile presented graphically and interpreted by analysis of variance and multiple linear regression. The apparent density, medium, maximum and minimum for particleboard obtained by microdensitometry X-ray ranged 357-697 $\mathrm{kg} / \mathrm{m}^{3}, 386-824 \mathrm{~kg} / \mathrm{m}^{3}$, and $334-634 \mathrm{~kg} / \mathrm{m}^{3}$, respectively, and gravimetric method $369-742 \mathrm{~kg} / \mathrm{m}^{3}$. The technique was effective in determining variations in the density profile in the thickness of the particleboards in

1 Engenheiro Florestal, Dr., Professor do Departamento de Ciências Florestais e da Madeira, Centro de Ciências Agrárias e Engenharias, Universidade Federal do Espírito Santo, Av. Governador Lindemberg, 316, Centro, CEP 29550-000, Jerônimo Monteiro (ES), Brasil. fabricio.goncalves@ufes.br

2 Engenheiro Florestal, Dr., Professor do Departamento de Produtos Florestais, Universidade Federal Rural do Rio de Janeiro, Antiga Estrada Rio-São Paulo, Km 47, CEP 23890-000, Seropédica (RJ), Brasil. lelis@ufrrj.br / amcarvalho@ ufrrj.br

3 Engenheiro Agrônomo, Dr., Professor do Departamento de Ciências Florestais, Escola Superior de Agricultura Luiz de Queiroz, Av. Pádua Dias, 11, Caixa Postal 09, CEP 13418-900, Piracicaba (SP), Brasil. mtomazel@usp.br

Recebido para publicação em 17/02/2016 e aceito em 6/07/2017

Ci. Fl., v. 28, n. 3, jul. - set., 2018 
all treatments, as well as direct obtain the apparent density. These variations could be reduced if the tannin used was previously hydrated in water. Was found strong correlation $\left(\mathrm{R}^{2}=0.99\right)$ between the mean apparent density obtained by X-ray microdensitometer and gravimetric method.

Keywords: quality panel; non-destructive analysis; density profile.

\section{INTRODUÇÃO}

Os painéis aglomerados são compósitos fabricados a partir de partículas de madeira seca, pulverizadas com um adesivo específico e aderidas pela aplicação de pressão e alta temperatura (KOLLMANN; KUENZI; STAMM, 1975; MALONEY, 1993). Podem ser formados exclusivamente com partículas de madeira de uma espécie ou de mistura de diversas espécies e, mesmo com outros componentes lignocelulósicos, resíduos madeireiros, mistura com plásticos, entre outros materiais.

Os painéis aglomerados são produzidos com base na razão de compactação de suas partículas, e sua qualidade, segundo Kollmann, Kuenzi e Stamm (1975), está diretamente associada à densidade da matéria-prima. Maloney (1993) menciona que uma razão de compactação ótima deve situar-se entre 1,3 e 1,6, para que desta forma, se possa garantir uma boa formação do painel. Ainda associado a isto estão os diferentes adesivos potenciais que podem ser empregados na sua manufatura, em função do destino final do produto. Estes adesivos podem ser de origem animal, sintéticos termorrígidos ou sintéticos termoplásticos. Entre os mais utilizados pela indústria de painéis aglomerados está a ureia formaldeído (MELO et al., 2010).

No entanto, devido a diversas circunstâncias, novas pesquisas vêm se destacando a fim de desenvolver adesivos eficientes na colagem e a menor custo, como aqueles à base de tanino, que pode ser usado na forma pura ou associado a diferentes adesivos químicos industriais (PIZZI, 2003; GONÇALVES; LELIS, 2009; GONÇALVES et al., 2016).

Considerando a qualidade dos painéis aglomerados produzidos para diferentes usos, a densidade aparente constitui-se uma das propriedades físicas mais importantes, sendo determinada em análises de rotina dos laboratórios e nas indústrias, por afetar outras propriedades físicas e mecânicas, além de estar relacionada com alguns parâmetros de processamento (pressão, temperatura, tempo), adesivo e madeira (espécie, geometria das partículas, etc.) (KELLY, 1977; MALONEY, 1993; NEMLI; DEMIREL, 2007). O perfil e a variação dos valores de densidade aparente ao longo da espessura dos painéis aglomerados de madeira são parâmetros importantes na avaliação das variáveis do processo e da matéria-prima, e determina a sua qualidade e uso final (WANG et al., 2006).

A aplicação de metodologia de análise e de obtenção do perfil da densidade aparente em painéis por microdensitometria de raios X tem sido relatada na literatura por alguns autores (BUCUR, 2003; WANG et al., 2006; TOMAZELLO FILHO et al., 2010), principalmente por ser uma técnica não destrutiva e de rápida obtenção de dados (GRECON, 2014).

O perfil de densidade aparente dos painéis de partículas de madeira, obtido pela densitometria de raios X, permite inferir sobre as diferentes etapas do processo, como nos parâmetros de fabricação do painel (teor de umidade, densidade da madeira, geometria das partículas), ciclo de prensagem no laboratório e na indústria (WANG; WINISTORFER; YOUNG, 2004; BELINI et al., 2012). Essa técnica possibilita, da mesma forma, analisar comparativamente painéis OSB, compensados, MDFs e painéis aglomerados, evidenciando a distribuição da densidade em vários pontos do painel. A qualidade do painel pode ser determinada pela técnica de raios X, horizontalmente e no perfil do painel, permitindo assim, mostrar a relação entre as propriedades do painel e a densidade aparente estimada (WANG; SALENIKOVICH; MOHAMMAD, 2007; CHEN et al., 2010; SURDI et al., 2014). A densitometria de raios X permite, ainda, a caracterização tecnológica detalhada da madeira, de painéis MDF, além da detecção de alterações anatômicas e físicas, como a induzida por fungos de podridão-branca e do efeito das práticas silviculturais na qualidade da madeira (TOMAZELLO FILHO et al., 2010).

O presente trabalho teve como objetivo apresentar a variação da densidade aparente de painéis aglomerados produzidos com partículas da madeira de Acacia mangium aplicando-se a metodologia de raios $\mathrm{X}$, em comparação ao método convencional gravimétrico. 


\section{MATERIAL E MÉTODOS}

\section{Origem da matéria-prima e confecção dos painéis aglomerados}

Foram confeccionados painéis aglomerados com a mistura de partículas da madeira de 10 árvores de Acacia mangium, com diâmetro a altura do peito entre $18-20 \mathrm{~cm}$. As árvores foram fornecidas pela empresa Cenibra S. A. e eram provenientes de uma plantação de 50 meses de idade, localizada no município de Santa Bárbara, estado de Minas Gerais. O local de plantio das árvores tem clima do tipo Cwb, segundo classificação climática de Köppen; temperatura média anual de $21,9^{\circ} \mathrm{C}$ e precipitação anual de $1.440 \mathrm{~mm}$. As árvores selecionadas foram provenientes de um plantio consorciado com Eucalyptus urophylla $\mathrm{x}$ Eucalyptus grandis, sendo que cada espécie ocupava 50\% da plantação. A madeira da A. mangium apresentava uma densidade básica média igual a $0,33 \mathrm{~g} / \mathrm{cm}^{3}$ (GONÇALVES; LELIS, 2012).

O adesivo utilizado foi a ureia formaldeído (nome comercial MDP1021), com teor de sólidos de $64 \%$, $\mathrm{pH}$ de 8,5 , viscosidade de $371,86 \mathrm{cP}$ e densidade de $1,23 \mathrm{~g} / \mathrm{cm}^{3}$. A proporção de adesivo ureia formaldeído utilizada na confecção dos painéis foi de $10 \%$ em relação à massa seca das partículas de madeira. $\mathrm{O}$ tanino em pó de Acacia mearnsii (nome comercial Phenothan AP) utilizado na confecção dos painéis aglomerados tinha $12 \%$ de umidade e foi aplicado na forma em pó às partículas após o encolamento com ureia formaldeído. Os painéis foram fabricados com tempo de prensagem de 8 minutos, temperatura de $140^{\circ} \mathrm{C}$ e pressão de 3,92 Mpa na dimensão nominal de $40 \times 40 \times 1,27 \mathrm{~cm}$ com quatro razões de compactação (1,39; 1,$55 ; 1,73$; e, 2,00) combinadas a quatro proporções de tanino em pó $(0,10,20$ e 30\%), totalizando 16 tratamentos (4 painéis por tratamento) (GONÇALVES, 2012) (Tabela 1).

TABELA 1: Descrição dos tratamentos utilizados na fabricação dos painéis aglomerados homogêneos.

TABLE 1: Description of treatments used in the manufacture of homogeneous particleboard.

\begin{tabular}{ccc}
\hline & \multicolumn{2}{c}{ Descrição } \\
\cline { 2 - 3 } Tratamento & Razão de & Proporção de Tanino em pó Aplicado às partículas \\
& compactação & previamente encoladas com ureia formaldeído \\
\hline T1 & & $0: 100$ \\
T2 & 1,39 & $10: 90$ \\
T3 & & $20: 80$ \\
T4 & & $30: 70$ \\
\hline T5 & & $0: 100$ \\
T6 & 1,55 & $10: 90$ \\
T7 & & $20: 80$ \\
T8 & & $30: 70$ \\
\hline T9 & & $0: 100$ \\
T10 & 1,73 & $10: 90$ \\
T11 & & $20: 80$ \\
T12 & & $30: 70$ \\
\hline T13 & & $0: 100$ \\
T14 & 2,00 & $10: 90$ \\
T15 & & $20: 80$ \\
T16 & & $30: 70$ \\
\hline
\end{tabular}




\section{Confecção dos corpos de prova dos painéis aglomerados}

Foram produzidos 64 painéis aglomerados homogêneos em relação ao tamanho e tipo de partícula ao longo de sua espessura e ou superfície, sendo retirados dois corpos de prova de 50 x $50 \mathrm{~mm}$ de cada painel, compreendendo 8 corpos de prova/tratamento. Destes, foram selecionados ao acaso 6 corpos de prova para as análises de microdensitometria e determinação da densidade aparente pelo método gravimétrico. Os 96 corpos de prova foram previamente acondicionados em sala de climatização por 72 horas, a temperatura do ar de $20^{\circ} \mathrm{C}$ e umidade relativa do ar de $60 \%$.

\section{Determinação da densidade aparente dos painéis aglomerados por microdensitometria de raios $\mathbf{X}$}

Os corpos de prova foram inseridos no porta-amostra (capacidade para doze amostras) e transferidos para o compartimento interno blindado de um microdensitômetro de raios X marca QMS modelo QDP-01X (Figura 1), com voltagem de 10-50 KV, corrente de 1,5 mA, colimação inicial e final dos feixes de 180 e de $90 \mu \mathrm{m}$, respectivamente.
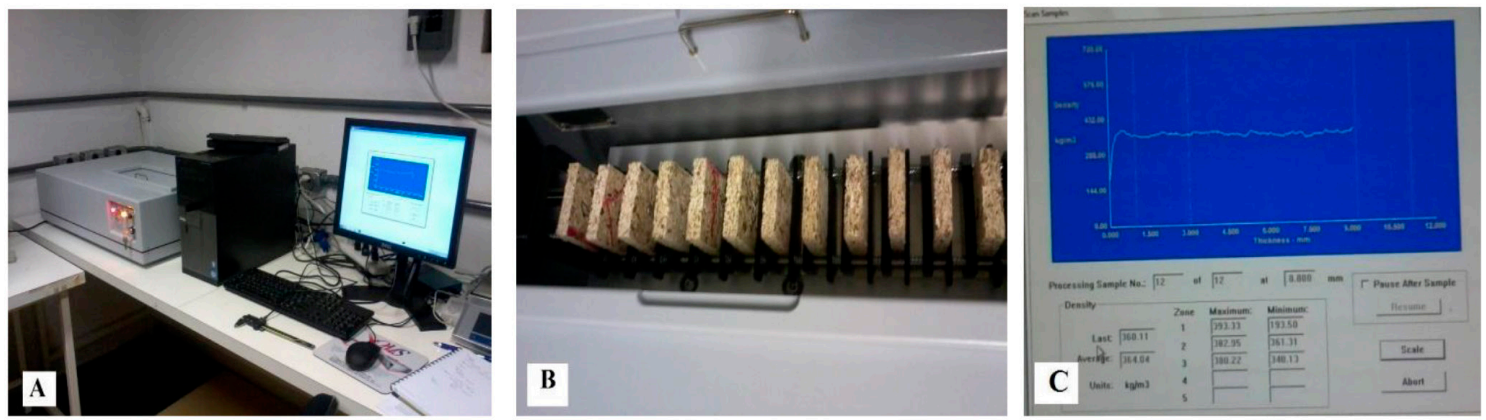

FIGURA 1: Obtenção do perfil de densidade aparente dos painéis aglomerados por microdensitometria de raios X. (A) equipamento microdensitômetro de raios $\mathrm{X}$, (B) porta-amostra com os corpos de prova dos painéis aglomerados $(50 \times 50 \mathrm{~mm})$ na câmara de irradiação do equipamento, (C) processo de varredura das amostras pelo feixe colimado de raios $\mathrm{X}$ e geração do respectivo perfil de densidade aparente do painel aglomerado.

FIGURE 1: Obtaining apparent density profile of particleboards by X-ray microdensitometry. (A) X-ray microdensitometry equipment, (B) sample holder with the specimens of particleboards $(50 \times 50 \mathrm{~mm})$ in the irradiation chamber, (C) scanning of the samples by X-ray collimated and generation density profile.

Inicialmente, o equipamento foi calibrado para operação de escaneamento (varredura) contínuo ao longo da espessura dos corpos de prova dos painéis aglomerados, pelos feixes colimados de raios X. O software controla o funcionamento da ampola de raios X, possibilitando a geração de um feixe de raios X estável que, em seguida, é colimado através da passagem por uma fenda de um bloco metálico $(0,005 \mathrm{~cm}$ de largura; $2,5 \mathrm{~cm}$ de altura) e, na sequência, a dose de raios $\mathrm{X}$ total incide sobre a amostra do painel aglomerado (comprimento de $50 \mathrm{~mm}$ ), sendo uma parte desta radiação atenuada. A radiação que atravessa o painel é analisada no detector de cristal, possibilitando a sua detecção e quantificação. 
A intensidade de raios $\mathrm{X}$ que atravessa pontualmente a amostra do painel, incide sobre o detector, sendo transformada em densidade aparente imediatamente por meio do software QMS, baseando-se nos seguintes parâmetros:

$$
\frac{\mathrm{I}}{\mathrm{I}_{0}}=\mathrm{e}^{-\mu_{1} \mathrm{t}}=\mathrm{e}^{-\mu_{\mathrm{m}} \rho \mathrm{t}}
$$

Em que: $\mathrm{I}=$ intensidade de radiação que transpassa o corpo de prova (total de fótons por unidade de área por unidade de tempo); $\mathrm{I}_{0}=$ intensidade de radiação que ficou retida no corpo de prova; $\mu_{1}=$ coeficiente de atenuação linear do material $\left(\mathrm{cm}^{-1}\right) ; \mu_{\mathrm{m}}=$ coeficiente de atenuação da massa do material $\left(\mathrm{cm}^{2} / \mathrm{g}\right) ; \rho=$ densidade do material $\left(\mathrm{g} / \mathrm{cm}^{3}\right) ; \mathrm{t}=$ espessura do corpo de prova; $e=$ logaritmo de base natural.

As seis amostras de painéis aglomerados inseridas no porta-amostra foram, desta forma, analisadas (irradiadas) em operação sequencial pelo feixe colimado de raios $\mathrm{X}$, com velocidade da varredura precisamente controlada, propiciando a leitura da densidade aparente em intervalo constante ( $40 \mu \mathrm{m}$ de distância linear) ao longo da espessura do mesmo (1,54 cm em média). Esta leitura permite a determinação da sua (i) densidade média, (ii) densidade máxima e (iii) densidade mínima.

Utilizaram-se as mesmas amostras na determinação da densidade aparente por gravimetria no microdensitômetro de raios $\mathrm{X}$.

\section{Determinação da densidade aparente dos painéis aglomerados por gravimetria}

A densidade gravimétrica dos corpos de prova dos painéis aglomerados foi determinada mediante obtenção de seu volume (comprimento, largura e espessura), obtidos com paquímetro digital de $0,1 \mathrm{~mm} \mathrm{e}$ de sua massa, por meio de uma balança digital de precisão de $0,001 \mathrm{~g}$. Ambos os equipamentos funcionavam acoplados diretamente ao microdensitômetro de raios X, consequentemente, ao computador e ao software de análise e os valores inseridos automaticamente no programa utilizado para geração das densidades.

\section{Análise estatística}

Aos resultados aplicou-se a análise de variância por meio do delineamento inteiramente casualizado e o teste de Scott-Knott, a 5\% de significância, para a comparação dos valores de densidade média, máxima, mínima e gravimétrica dos painéis aglomerados. Os valores de densidade média, máxima mínima e gravimétrica foram submetidos aos testes de normalidade de Kolmogorov-Smirnov, Cramér-von Mises e Watson, todos ao nível de significância de 5\% e também ao teste de homogeneidade de variância de Bartlett.

A análise de regressão linear múltipla foi realizada com o objetivo de relacionar as densidades média máxima e média mínima em relação à densidade média do painel, obtidas por densitometria de raios $\mathrm{X}$ mediante o modelo matemático abaixo:

$$
Y_{i}=\beta_{0}+\beta_{1} X_{1 i}+\beta_{2} X_{2 i}+e_{i}
$$

Em que: $Y_{\mathrm{i}}=$ Densidade aparente média dos painéis, em $\mathrm{kg} / \mathrm{m}^{3} ; \mathrm{X}_{1 \mathrm{i}}=$ Densidade aparente máxima média, em $\mathrm{kg} / \mathrm{m}^{3}$; $\mathrm{X}_{2 \mathrm{i}}=$ Densidade aparente mínima média, em $\mathrm{kg} / \mathrm{m}^{3}$.

\section{RESULTADOS E DISCUSSÃO}

\section{Densidade aparente dos painéis}

Os valores de densidade aparente dos painéis aglomerados de partículas de madeira de Acacia mangium determinados pela metodologia de microdensitometria de raios $\mathrm{X}$ e pelo método gravimétrico mostram variações significativas entre os tratamentos (Tabela 2). 
TABELA 2: Valores médios de densidade aparente determinada por microdensitometria de raios $\mathrm{X}$ e por método gravimetria dos painéis de partículas de madeira de Acacia mangium.

TABLE 2: Average values of apparent density by X-ray microdensitometry and gravimetric method in the particleboards of Acacia mangium wood.

\begin{tabular}{|c|c|c|c|c|}
\hline \multirow{3}{*}{ Tratamento } & \multirow{2}{*}{\multicolumn{3}{|c|}{$\begin{array}{c}\text { Densidade aparente dos painéis }\left(\mathrm{kg} / \mathrm{m}^{3}\right) \text { por } \\
\text { Microdensitometria de raios } \mathrm{X}\end{array}$}} & \multirow{3}{*}{$\begin{array}{l}\text { Gravimetria } \\
\text { Média } \\
\left(\mathrm{kg} / \mathrm{m}^{3}\right)\end{array}$} \\
\hline & & & & \\
\hline & Média & Máxima & Mínima & \\
\hline $\mathrm{T} 1$ & $450 \pm 45 \mathrm{a}$ & $510 \pm 72 \mathrm{a}$ & $420 \pm 40 \mathrm{a}$ & $460 \pm 41 \mathrm{a}$ \\
\hline $\mathrm{T} 2$ & $420 \pm 36 a$ & $470 \pm 44 \mathrm{a}$ & $390 \pm 35 a$ & $440 \pm 35 a$ \\
\hline $\mathrm{T} 3$ & $470 \pm 84 \mathrm{a}$ & $520 \pm 11 \mathrm{a}$ & $430 \pm 76 a$ & $480 \pm 88 a$ \\
\hline $\mathrm{T} 4$ & $440 \pm 61 \mathrm{a}$ & $480 \pm 65 \mathrm{a}$ & $410 \pm 54 \mathrm{a}$ & $450 \pm 64 \mathrm{a}$ \\
\hline T5 & $450 \pm 38 \mathrm{a}$ & $510 \pm 62 \mathrm{a}$ & $410 \pm 37 a$ & $460 \pm 37 \mathrm{a}$ \\
\hline T6 & $470 \pm 49 \mathrm{a}$ & $530 \pm 50 \mathrm{a}$ & $430 \pm 47 \mathrm{a}$ & $500 \pm 53 \mathrm{a}$ \\
\hline $\mathrm{T} 7$ & $450 \pm 51 \mathrm{a}$ & $520 \pm 65 \mathrm{a}$ & $370 \pm 12 \mathrm{a}$ & $470 \pm 57 \mathrm{a}$ \\
\hline $\mathrm{T} 8$ & $550 \pm 49 \mathrm{~b}$ & $640 \pm 72 b$ & $490 \pm 40 \mathrm{~b}$ & $570 \pm 50 b$ \\
\hline T9 & $510 \pm 40 \mathrm{~b}$ & $590 \pm 46 \mathrm{a}$ & $470 \pm 40 \mathrm{~b}$ & $540 \pm 41 b$ \\
\hline $\mathrm{T} 10$ & $540 \pm 53 b$ & $630 \pm 70 b$ & $490 \pm 50 \mathrm{~b}$ & $560 \pm 52 b$ \\
\hline T11 & $530 \pm 50 \mathrm{~b}$ & $630 \pm 71 \mathrm{~b}$ & $480 \pm 45 b$ & $570 \pm 55 b$ \\
\hline $\mathrm{T} 12$ & $470 \pm 65 \mathrm{a}$ & $540 \pm 79 \mathrm{a}$ & $420 \pm 50 \mathrm{a}$ & $490 \pm 65 a$ \\
\hline $\mathrm{T} 13$ & $570 \pm 46 b$ & $670 \pm 65 b$ & $500 \pm 49 \mathrm{~b}$ & $600 \pm 50 \mathrm{~b}$ \\
\hline $\mathrm{T} 14$ & $610 \pm 58 \mathrm{c}$ & $720 \pm 54 \mathrm{c}$ & $560 \pm 52 \mathrm{~b}$ & $650 \pm 59 c$ \\
\hline $\mathrm{T} 15$ & $620 \pm 46 c$ & $750 \pm 54 \mathrm{c}$ & $550 \pm 55 \mathrm{~b}$ & $660 \pm 50 \mathrm{c}$ \\
\hline $\mathrm{T} 16$ & $590 \pm 91 \mathrm{c}$ & $690 \pm 11 \mathrm{c}$ & $520 \pm 83 \mathrm{~b}$ & $610 \pm 88 c$ \\
\hline
\end{tabular}

$\mathrm{Na}$ coluna, letras iguais não diferem estatisticamente pelo teste de Scott-Knott $(\mathrm{p}<0,05)$. Valor após a média corresponde ao desvio padrão.

Destacam-se os tratamentos de confecção dos painéis aglomerados que aplicam as razões de compactação mais elevadas, com os baixos valores dos desvios, indicando certa homogeneidade entre os tratamentos. No entanto, há diferenças significativas entre os tratamentos de confecção dos painéis que aplicam a mesma razão de compactação, demonstrando haver variáveis não controladas no processo produtivo. De uma forma geral, os valores obtidos por ambos os métodos se mostraram muito próximos do valor da densidade nominal para os painéis, o que de acordo com Trianoski et al. (2013), pode ser atribuído à perda de material durante a formação do painel e retorno em espessura após prensagem.

Os valores de densidade submetidos aos testes de normalidade e homogeneidade de variâncias apresentaram distribuição normal e com variâncias homogêneas e significativas. 
Os maiores valores absolutos de densidade aparente estão nos tratamentos de maior razão de compactação $(1,73$ e 2,00). A razão de compactação é obtida com base na relação entre a densidade do painel e a densidade básica da madeira (MOSLEMI, 1974; MALONEY, 1993); por este motivo, esperava-se que, conforme afirmado por Naumann et al. (2008), em maiores valores desta razão, houvesse uma chapa mais homogênea na mesma faixa de compactação, o que acabou não ocorrendo neste estudo. Outros fatores também podem contribuir na heterogeneidade dos painéis, como a geometria das partículas utilizadas, a acidez e teor dos extrativos da madeira, a quantidade e qualidade do adesivo utilizado, a pressão, temperatura, tempo de fechamento da prensa e tempo de prensagem (MOSLEMI, 1974; KELLY, 1977).

As condições laboratoriais de confecção, levando-se em conta a presença do tanino em pó na encoladeira e a condição da prensa, provavelmente foram responsáveis pela ocorrência de diferenças estatísticas significativas na densidade dos painéis aglomerados entre os tratamentos aplicados. Pesquisas realizadas em laboratórios normalmente trabalham com valores de densidades dos painéis próximos a $700 \mathrm{~kg} / \mathrm{m}^{3}$ (TOSTES et al., 2004; BELINI; TOMAZELLO FILHO; CHAGAS, 2009), assim como nas indústrias, sendo raramente encontrados painéis com densidade inferiores a $500 \mathrm{~kg} / \mathrm{m}^{3}$.

Nemli e Demirel (2007) encontraram uma densidade média de $900 \mathrm{~kg} / \mathrm{m}^{3}$ em painel multicamada, confeccionado com quatro espécies, sendo elas Fagus orientalis, Populus tremula, Pinus sylvestris e Quercus cerris, em prensa estacionária. Os autores ainda afirmaram que este tipo de orientação das partículas slivers proporciona um decréscimo da densidade do centro do painel devido à diminuição do número de partículas, com consequente aumento da densidade em ambas as superfícies.

No presente estudo, a forma de distribuição das partículas no colchão, por ser manual, e a velocidade de fechamento não controlada da prensagem podem ter influenciado na formação da densidade do painel, conforme já relatado por Matos (1988), uma vez que o mecanismo de ação do tempo de fechamento sobre a consolidação do colchão de partículas está associado à uniformidade de transferência de pressão e calor às camadas interiores.

Em um rápido tempo de fechamento as camadas superficiais densificam mais rapidamente, não ocorrendo uma compressão uniforme através do material, pois com o miolo ainda frio há uma maior resistência à compactação nestas camadas. Logo, o gradiente vertical de densidade é mais pronunciado com grande densificação nas camadas superficiais e densidades menores no miolo. Por outro lado, para um tempo de fechamento mais lento ocorre uma distribuição mais uniforme da densificação das partículas devido à compressão gradual associada com a entrada de calor nas camadas interiores (MATOS, 1988).

Chen et al. (2010) estudando a variação da densidade por raios X em diferentes tipos de painéis comerciais (fibras de média densidade, aglomerado, partículas orientadas e compensado), encontraram valores médios da ordem de $690 \mathrm{~kg} / \mathrm{m}^{3}$; e confirmaram também a existência de variações de densidades ao longo do comprimento dos painéis.

\section{Perfis de densidade aparente}

A Figura 2 mostra os perfis médios obtidos nos painéis aglomerados, provenientes de seis amostras de cada tratamento estudado. 

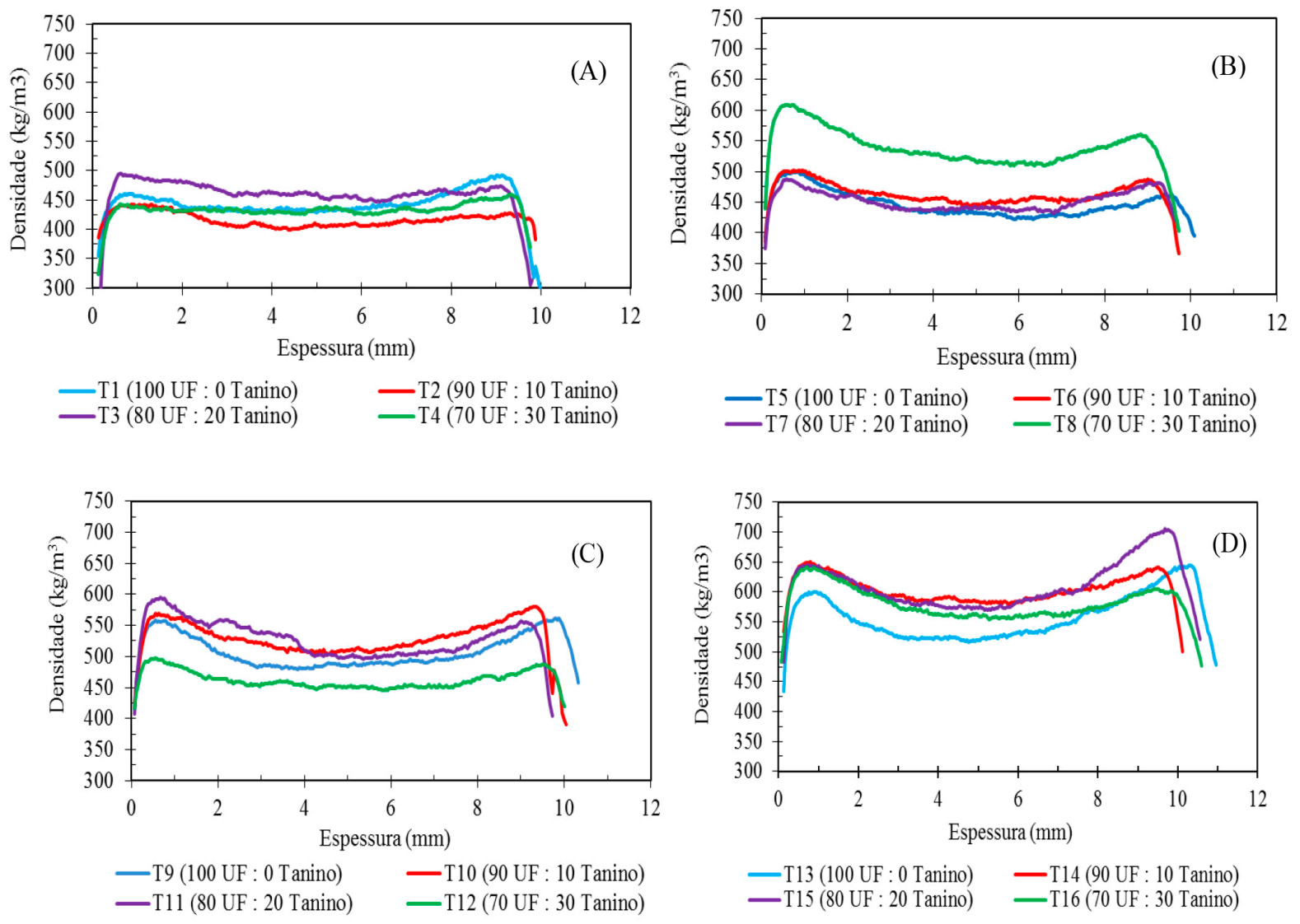

FIGURA 2: Perfil de densidade aparente média dos painéis aglomerados na razão de compactação de 1,39 (A), 1,55 (B), 1,73 (C) e 2,00 (D).

FIGURE 2: Apparent density profiles mean of the particleboard in the compression ratio of 1.39 (A), 1.55 (B), 1.73 (C) and $2.00(\mathrm{D})$.

Independentemente da razão de compactação e do teor de tanino em pó aplicado às partículas encoladas com ureia formaldeído, as maiores densidades foram registradas nas faces superior e inferior dos painéis, com redução para o centro dos mesmos, sendo mais homogêneo ao longo do perfil na menor razão de compactação. Este comportamento geral pode ser devido à baixa aderência do tanino em pó e sua solubilidade diretamente em contato com as partículas já encoladas sendo acamado sobretudo em uma das faces, como observado na Figura 2, em especial nos tratamentos T1, T8, T11, T15.

A avaliação dos valores de densidade média, mínima e máxima (Tabela 1) e do perfil de densidade ao longo da espessura dos painéis aglomerados de Acacia mangium, avaliados por microdensitometria de raios $\mathrm{X}$, indicam haver regiões com densidades similares, independentemente dos tratamentos utilizados, sobretudo naqueles de menor razão de compactação (Figura 2A), observando-se também menor variação da densidade na parte central dos painéis. A adição de tanino em pó às partículas fez com que o perfil de densidade fosse alterado em praticamente todas as razões de compactação, contribuindo para um aumento da densidade (Tabela 1 e Figura 2). Este comportamento foi melhor observado nas maiores adições de tanino, com melhor destaque para a razão de compactação de 2,00 (Figura 2D). Isso pode ser explicado devido à maior densificação das partículas, mesmo observando-se diferenças estatísticas significativas entre os tratamentos.

O perfil de densidade descreve a densidade do painel na camada de partículas que o compõe ao longo de sua espessura. O perfil em forma de "M" caracteriza superfícies de alta densidade nas extremidades com o seu centro de menor densidade, o que é normalmente formado em painéis prensados a quente, podendo afetar quase todas as propriedades físicas e mecânicas dos painéis (XU, 1999; WONG et al., 2000).

$\mathrm{O}$ perfil em "M", característicos nos painéis MDF confeccionados com madeira de eucalipto e 
outras espécies (TOMAZELLO FILHO et al., 2010; RAUTKARI; KAMKE; HUGHES, 2011) não foi observado nitidamente em qualquer das razões de compactação. No entanto, nota-se uma tendência deste comportamento na razão de compactação de 2,00 (Figura 2D). A adição de $30 \%$ de tanino na razão de compactação de 1,55 e a adição de $20 \%$ de tanino na razão de compactação de 2,00 proporcionou pico de densidade na extremidade dos painéis. Este comportamento está associado à sua dificuldade de ligação com a ureia formaldeído contida na partícula, tendo o mesmo sido deslocado para a extremidade do painel no momento da confecção do colchão.

O modelo de perfis de densidade dos painéis aglomerados parece estar relacionado com as características das partículas da madeira de Acacia mangium, uma vez que as mesmas apresentaram-se com certa lanosidade (que corresponde a uma superfície macia, desenvolvida quando a madeira exibe fibras individuais ou em pequenos grupos de forma solta), assim como a maior quantidade aplicada de tanino em pó, após o encolamento dos painéis com ureia formaldeído, propiciando um perfil de densidade aparente mais uniforme ao longo da espessura.

Com a adição de $30 \%$ de tanino em pó às partículas ocorreu um aumento da densidade aparente média (Figura 2B), o qual foi proporcionado principalmente em função dos sólidos presentes no adesivo. Comportamento distinto foi observado nas outras razões de compactação para a mesma quantidade de tanino.

Na razão de compactação 1,73 (Figura 2C) o perfil em forma de "M" apresenta-se bem pronunciado nas situações onde foi aplicado o tanino às partículas - 10 e $20 \%$ de tanino. Nesta razão de compactação, a adição de 30\% de tanino apresentou um perfil de densidade aparente inferior em relação as demais razões de compactação. Possivelmente ocorreu um efeito não controlado quando na formação do painel.

A maior razão de compactação proporcionada aos painéis aglomerados (Figura 2D) produziu perfis de densidade mais homogêneos, sem a presença de picos pronunciados ao longo do perfil, para todos os tratamentos. Observando-se a Tabela 1 pode-se constatar que a razão de compactação igual a 2,00 manteve a variação em $9,8 \%, 10,1 \%, 10,2 \%$ e $11,3 \%$, respectivamente, para as densidades média, máxima, mínima e gravimétrica.

A propriedade do painel com $100 \%$ de ureia formaldeído a uma razão de compactação igual a 2,00 apresentou-se diferentemente dos demais, atingindo uma menor densidade em uma das extremidades e no miolo do painel.

Para aquelas situações em que foi utilizada somente a resina ureia formaldeído, em especial nas razões de compactação de 1,73 e 2,00, apesar de não haver diferenças estatísticas entre alguns tratamentos, o comportamento do perfil da densidade (Figuras 2C e 2D) foi mais uniforme; o mesmo não foi observado para os demais tratamentos com adição de tanino. As variações ocorridas nos perfis de densidade podem estar associadas à inconsistência do encolamento das partículas e ainda durante a formação do colchão (CHEN et al., 2010).

\section{Correlação entre as densidades}

A Figura 3 mostra uma forte correlação da densidade aparente média obtida por microdensitometria de raios X com a medição gravimétrica, expressada pelo forte valor do coeficiente de determinação de $0,99(\mathrm{p}<0,05)$. O presente estudo encontrou o mesmo valor absoluto para o coeficiente de determinação do observado por Wang et al. (2006), indicando ser a técnica de raios X bastante confiável e precisa para aplicação neste tipo de painel, sobretudo na espessura estudada. 


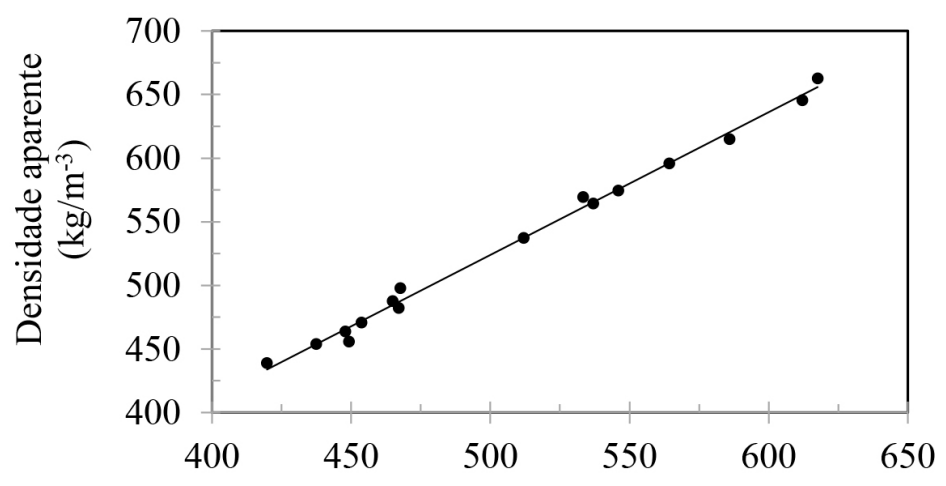

Densidade gravimétrica $\left(\mathrm{kg} / \mathrm{m}^{-3}\right)$

FIGURA 3: Correlação da densidade aparente média dos painéis aglomerados de madeira de Acacia mangium por microdensitometria de raios $\mathrm{X}$ e por gravimetria.

FIGURE 3: Correlation of average apparent density of particleboards of Acacia mangium by X-ray microdensitometry and gravimetry.

Linville (2000) registrou um coeficiente de determinação de 0,90 entre ambas as densidades máximas e mínimas trabalhando com painel OSB de madeira de Populus sp. com adesivo de base MDI (Difenil Metano Di-Isocianato) em três proporções diferentes. Apesar de ser mais poroso e haver maior variação de densidade no interior deste tipo de painel, a dispersão dos dados encontrada pelo autor, representa a distribuição dos erros residuais, com um desvio padrão constante.

\section{Valores de densidades média, máxima e mínima}

A análise de regressão linear múltipla indicativa da relação da densidade aparente média e a densidade máxima e mínima e sua ordem de importância, considerando o valor médio dos 96 corpos de prova dos painéis aglomerados apresentou, por meio da regressão $(\mathrm{p}<0,01$ pelo teste $t)$ que as duas densidades extremas (máxima e mínima) do modelo de regressão explicam $97,3 \%$ da variação total:

$$
\hat{\mathrm{D}}_{\text {média }}=0,05958+0,61468^{* *} \mathrm{D}_{\text {máximo }}+0,18908^{* *} \mathrm{D}_{\text {mínimo }}
$$

Isso indica também que as densidades máximas e mínimas explicam 85,24 e 21,40\% da variação total da densidade, respectivamente. Ambas as densidades foram significativas e, portanto, mantidas na equação. A importância de contribuição de cada uma das variáveis $D_{i}(i=1,2)$ foi medida com base no coeficiente de determinação parcial, conforme Hoffmann e Vieira (1983). A importância da contribuição de $\mathrm{D}_{\text {máxima }}\left(\mathrm{X}_{1}\right)$ e $\mathrm{D}_{\text {mínima }}\left(\mathrm{X}_{2}\right)$ é demonstrada a seguir:

$$
\begin{aligned}
& \mathrm{R}_{\mathrm{YX}_{1}, \mathrm{X}_{2}}^{2}=\frac{\mathrm{R}^{2}-\mathrm{R}_{\mathrm{YX}_{2}}^{2}}{1-\mathrm{R}_{\mathrm{YX}_{2}}^{2}}=\frac{0,9727-0,8150}{1-0,8150}=0,8524^{* *} \\
& \mathrm{R}_{\mathrm{YX}_{2}, \mathrm{X}_{1}}^{2}=\frac{\mathrm{R}^{2}-\mathrm{R}_{\mathrm{YX}_{1}}^{2}}{1-\mathrm{R}_{\mathrm{YX}_{1}}^{2}}=\frac{0,9727-(0,98247)^{2}}{1-(0,98247)^{2}}=0,2140^{* *}
\end{aligned}
$$

A explicação de quanto uma variável influencia na resposta de outra é muito utilizada por alguns autores mediante o programa Stepwise, quando há a existência de muitas variáveis (ELEOTÉRIO; TOMAZELLO FILHO; BORTOLETTO JUNIOR, 2000; ROQUE; TOMAZELO FILHO, 2007), sendo um procedimento de inclusão ou exclusão de alguma ou algumas variáveis de resposta, facilitando assim uma melhor interpretação dos resultados. A definição da melhor resposta pode ser adotada com base no melhor coeficiente de determinação. Porém, como neste estudo somente foram avaliadas duas variáveis de resposta (densidade máxima e densidade mínima), optou-se por não retirar uma ou outra variável. Assim, os coe- 
ficientes de correlação obtidos apresentaram-se significativos, independentemente da relação de uma ou outra variável de resposta.

\section{CONCLUSÃO}

A técnica de microdensitometria de raios X com obtenção direta da densidade aparente possui grande capacidade de exploração e geração de dados dentro de uma avaliação não destrutiva na avaliação de painéis aglomerados, a qual permitiu apurar o perfil da densidade dos mesmos.

Os perfis densitométricos obtidos ao longo da espessura dos painéis pela microdensitometria de raios $\mathrm{X}$ mostraram significativas variações da estrutura dos mesmos.

Uma hidratação no tanino em pó permitiria uma melhor aderência à resina ureia-formaldeído, melhorando as pequenas variações de densidade observadas no perfil densitométrico.

A técnica permitiu observar a existência de menores valores absolutos médios quando comparada à técnica de obtenção de densidade por gravimetria.

A correlação entre a densidade aparente e a densidade gravimétrica indica que ambas as densidades médias se correlacionam entre si.

\section{REFERÊNCIA}

BELINI, U. L. et al. Teor de sílica em compósitos confeccionados com bagaço de cana-de-açúcar e eucalipto. Floresta e Ambiente, Seropédica, v. 19, n. 2, p. 250-255, jun. 2012.

BELINI, U. L.; TOMAZELLO FILHO, M.; CHAGAS, M. P. Densitometria de raios X aplicada na avaliação tecnológica de painéis MDF de eucalipto. Scientia Forestalis, Piracicaba, v. 37, n. 84, p. 343-350, dez. 2009.

BUCUR, V. Techniques for high resolution imaging of wood structure: a review. Measurement Science and Technology, Bristol, v. 14, n. 12, p. 91-98, oct. 2003.

CHEN, S. et al. Digital X-ray analysis of density distribution characteristics of wood-based panels. Wood Science and Technology, Berlin, v. 44, n. 1, p. 85-93, may 2010.

ELEOTÉRIO, J. R.; TOMAZELLO FILHO, M.; BORTOLETTO JUNIOR, G. Propriedades físicas e mecânicas de painéis MDF de diferentes massas específicas e teores de resina. Ciência Florestal, Santa Maria, v. 10, n. 2, p. 75-90, 2000.

GONÇALVES, F. G. Painéis aglomerados de madeira de Acacia mangium com adesivos de uréia-formaldeído e tanino em pó da casca de Acacia mearnsii. 2012, 105 f. Tese (Doutorado) - Universidade Federal Rural do Rio de Janeiro, Seropédica, 2012.

GONÇALVES, F. G.; LELIS, R. C. C. Caracterização Tecnológica da Madeira de Acacia mangium Willd em Plantio Consorciado com Eucalipto. Floresta e Ambiente, Seropédica, v. 19, n. 3, p. 286-295, 2012.

GONÇALVES, F. G. et al. Avaliação da resistência ao cisalhamento da madeira de Pinus sp. coladas em temperatura ambiente. Ciência da Madeira, Pelotas, v. 7, n. 1, p. 42-50, abr. 2016.

GONÇALVES, F. G.; LELIS, R. C. C. Propriedades de duas resinas sintéticas após adição de tanino modificado. Floresta e Ambiente, Seropédica, v. 16, n. 2, p. 1-7, 2009.

GRECON. Stenograph. 2014. Disponível em: $<$ http://www.grecon.com/pt/stenograph $>$. Acesso em: 17 abr. 2014.

HOFFMANN, R.; VIEIRA, S. Análise de regressão: uma introdução à econometria. 2. ed. São Paulo: HUCITEC, 1983. 379 p.

KELLY, M. W. Critical literature review of relationship between processing parameters and physical properties of particleboard. Gen. Tech. Rep. FPL-10, Madison: USDA Forest Service, Forest Products Laboratory, 1977. 70 p.

KOLLMANN, F. F. P.; KUENZI, E. W.; STAMM, A. J. Principles of wood science and technology: wood based materials. Berlin: Springer-Verlog, 1975. v. 2. 694 p.

LINVILLE, J. D. The influence of a horizontal density distribution on moisture-related mechanical degradation of oriented strand composites. 2000. $122 \mathrm{f}$. Thesis (Master of Science in Civil Engineering) 
- Washington State University, Washington, 2000.

MALONEY, T. M. Modern particleboard and dry-process fiberboard manufacturing. San Francisco: M. Freeman, 1993. 688 p.

MELO, R. R. et al. Resistência de painéis aglomerados produzidos com diferentes proporções de madeira e casca de arroz a fungos e cupins xilófagos. Ciência Florestal, Santa Maria, v. 20, n. 3, p. 501-511, jul./ set. 2010.

MOSLEMI, A. A. Particleboard. Carbondele: Southern Illinois Press, 1974. v.1. 256 p.

MATOS, J. L. M. Ciclo da Prensa em Chapas de Partículas Estruturais "Waferboard". 1988. $190 \mathrm{f}$. Dissertação (Mestrado) - Universidade Federal do Paraná, Curitiba, 1988.

NAUMANN, R. B. et al. Propriedades de chapas fabricadas com partículas de madeira de Eucalyptus urophylla S. T. Blake e de Schizolobium amazonicum Herb. Revista Árvore, Viçosa, MG, v. 32, n. 6, p. 1143-1150, dez. 2008.

NEMLI, G.; DEMIREL, S. Relationship between the density profile and the technological properties of the particleboard composite. Journal of Composite Materials, Lancaster, v. 41, n. 15, p. 1793-1802, aug. 2007.

PIZZI, A. Natural Phenolic Adhesives I: Tannin. In: PIZZI, A.; MITTAL, K. L. (Ed.). Handbook of Adhesive Technology. 2 nd ed. rev. exp. Madison: Marcel Dekker, 2003. Cap. 27.

RAUTKARI, L.; KAMKE, F. A.; HUGHES, M. Potential error in density profile measurements for wood composites. European Journal of Wood and Wood Products, Berlin, v. 69, n. 1, p. 167-169, feb. 2011.

ROQUE, R. M.; TOMAZELO FILHO, M. Relationships between anatomical features and intra-ring wood density profiles in Gmelina arborea applying X-ray densitometry. Cerne, Lavras, v. 13, n. 4, p. 384-392, sep. 2007.

SURDI, P. G. et al. Relação entre perfil de densidade e ligação interna de painéis OSB de Pinus spp. Floresta e Ambiente, Seropédica, v. 21, n. 3, p. 349-357, jul./set. 2014.

TOMAZELLO FILHO, M. et al. Avaliação tecnológica da madeira e de painéis MDF de eucalipto por densitometria de raios X. Madeira: Arquitetura \& Engenharia, São Carlos, v. 11, n. 27, p. 45-58, dez. 2010. TOSTES, A. S. et al. Colagem de chapas de madeira aglomerada com adesivo uréia-formaldeído (UF) modificado com tanino da casca de Eucalyptus pellita F. Muell. Floresta e Ambiente, Seropédica, v. 11, n. 2, p. 14-19, dez. 2004.

TRIANOSKI, R. et al. Utilização da madeira de Cryptomeria japonica para produção de painéis aglomerados. Scientia Forestalis, Piracicaba, v. 41, n. 97, p. 57-64, mar. 2013

WANG, S.; WINISTORFER, P. M.; YOUNG, T. M. Fundamental of vertical density profile formation in wood composites: part 3- MDF density formation during hot pressing. Wood and Fiber Science, Madison, v. 36, n. 1, p. 17-25, jan. 2004.

WANG, X. et al. Evaluation of density distribution in wood-based panels using X-ray scanning. The eJournal of Nondestructive Testing, [s. 1.], v. 11, n. 4, apr. 2006.

WANG, X.; SALENIKOVICH, A.; MOHAMMAD, M. Localized density effects on fastener holding capacities in wood based panels. Forest Products Journal, Madison, v. 57, n. 1/2, p. 103-109, dec. 2007.

WONG, E. D. et al. Formation of the density profile and its effects on the properties of fiberboard. Journal of Wood Science, [s. 1.], v. 46, n. 3, p. 202-209, jun. 2000.

$\mathrm{XU}, \mathrm{W}$. Influence of vertical density distribution on bending modulus of elasticity of wood composite panels: a theoretical consideration. Wood and Fiber Science, Madison, v. 31, n. 3, p. 277-282, jul. 1999. 